\title{
Finding Non-Polynomial Positive Invariants and Lyapunov Functions for Polynomial Systems through Darboux Polynomials.
}

\author{
Eric Goubault*, Jacques-Henri Jourdan ${ }^{\dagger}$, Sylvie Putot* and Sriram Sankaranarayanan ${ }^{\ddagger}$ \\ ${ }^{*}$ CEA LIST, Nanoinnov, Gif-sur-Yvette, France Email: surname.name@cea.fr \\ †INRIA Rocquencourt, Rocquencourt, France Email: first.lastname@inria.fr \\ $\ddagger$ University of Colorado, Boulder, CO USA Email: first.lastname@colorado.edu
}

\begin{abstract}
In this paper, we focus on finding positive invariants and Lyapunov functions to establish reachability and stability properties, respectively, of polynomial ordinary differential equations (ODEs). In general, the search for such functions is a hard problem. As a result, numerous techniques have been developed to search for polynomial differential variants that yield positive invariants and polynomial Lyapunov functions that prove stability, for systems defined by polynomial differential equations. However, the systematic search for non-polynomial functions is considered a much harder problem, and has received much less attention.

In this paper, we combine ideas from computer algebra with the Sum-Of-Squares (SOS) relaxation for polynomial positive semi-definiteness to find non polynomial differential variants and Lyapunov functions for polynomial ODEs. Using the wellknown concept of Darboux polynomials, we show how Darboux polynomials can, in many instances, naturally lead to specific forms of Lyapunov functions that involve rational function, logarithmic and exponential terms. We demonstrate the value of our approach by deriving non-polynomial Lyapunov functions for numerical examples drawn from the literature.
\end{abstract}

\section{INTRODUCTION}

Positive invariants and Lyapunov functions are important tools for analyzing the reachability and stability of control systems modeled as Ordinary Differential Equations (ODEs). In this paper, we focus on techniques for automatically discovering non-polynomial functions in the form of differential variants to establish positive invariants, and Lyapunov functions to establish asymptotic stability of polynomial differential equations.

Our approach, in this paper, has been inspired by the study of integrability properties of polynomial dynamical systems [1] and related techniques used in formal integration to automatically derive first integrals of differential equations [2]. Starting with the well known concept of a Darboux polynomial, we show how the existence of Darboux polynomials can, in turn, lead to the existence of rational and logarithmic functions whose derivatives are positive definite. Our results lead to heuristic search procedures that extend well-known techniques for finding polynomial Lyapunov functions using sum-of-squares (SOS) reduction, to naturally discover rational function and sometimes transcendental functions with positive definite derivatives. Such functions are called differential variants, and are useful in finding positive invariants. We present techniques to find Lyapunov functions given such differential variants. To summarize the main contributions of this paper:
- Our results in Section III tie the existence of rational function differential variants (of the form $\frac{p(x)}{q(x)}$ for polynomials $p, q$ ) to that of Darboux polynomials under some assumptions about the structure of their differential remainder (Theorem 2). We also show a class reduction theorem that ties the existence of differential variants over the algebraic extensions to rational functions back to rational function differential variants (Theorem 3).

- We observe that the search for rational function differential variants can be automated by searching for a set of Darboux polynomials and setting up a search as an SOS programming problem (Section III-B).

- We investigate transcendental extensions to find differential variants involving logarithmic and exponential functions (Section IV).

- Finally, we extend our techniques for differential variants to also find Lyapunov functions (Section V).

We illustrate our technique by showing its ability to find rational function and logarithmic Lyapunov functions on some systems where such functions are known to exist in the related work (Section VI).

\section{A. Related Work}

The problem of automatically discovering Lyapunov functions has been well studied. For linear systems, it is possible to synthesize Lyapunov functions by solving Linear Matrix Inequalities (LMIs) [3]. However, the discovery of Lyapunov functions for non-linear systems has proved to be much harder in practice. The use of LMIs to find Lyapunov functions for non-linear systems has been studied by Tibken [4]. However, it has been observed that the synthesis of rational function invariants will involve bilinear or polynomial matrix inequalities (BMIs and PMIs) rather than LMIs. While techniques for solving BMIs have been well studied, solving them is computationally more expensive than LMIs [5]. This is why some authors have proposed other methods for determining a region of attraction than using Lyapunov functions, see e.g. [6].

The problem of automating Lyapunov function synthesis for non-linear systems has been studied by many. Notable contributions include Zubov's method which uses a partial differential equation to find a maximal region of attraction and an associated Lyapunov function [7]. A related approach is proposed by Vannelli and Vidyasagar discovering maximal 
region of attraction through rational Lyapunov functions [8]. Our approach to rational functions differs in many ways. We focus on rational functions whose Lie derivatives are a positive definite polynomial, whereas their work focuses on functions whose derivatives are a positive definite quadratic form plus a higher order rational function. Furthermore, we relate the existence of rational functions with Darboux polynomials, and rely on sum of squares optimization [9]. Vannelli and Vidyasagar, present an iterative linear algebra method based on repeated equation solving to discover the rational function.

Sum-Of-Squares (SOS) has proved to be an important tool for investigating nonlinear systems [9], [10]. The approach reduces polynomial positivity to a semi-definite program (SDP), using numerical solvers to find feasible solutions for the SDP. SOS was extended by Papachristadoulou and Prajna to non-polynomial systems by adding auxiliary variables to encode transcendental functions such as $\sin (z), \cos (z)$ and $\exp z$, where $z$ is a polynomial over the state variables [11].

Differential variants refer to functions $f$ whose derivatives are strictly positive, thus proving positive invariance of the set $\{\vec{x} \mid f(\vec{x}) \geq 0\}$. They underlie the study of invariants to prove reachability properties. The automatic generation of such functions has been studied by many including Tiwari et al. [12] and Platzer et al. [13]. Differential variants can be seen as a simplification of barrier functions studied by Prajna and Jadbabaie, who use sum of squares programming to automatically compute them for differential equations [14].

\section{Preliminaries}

In this section, we discuss some preliminary concepts including differential variants, Lyapunov functions, Darboux polynomials and some existing approaches to determine Darboux polynomials. Let $\mathbb{R}\left[x_{1}, \ldots, x_{n}\right]$ denote the set of polynomials over variables $x_{1}, \ldots, x_{n}$ and coefficients drawn from the reals. Likewise, let

$\mathbf{R}\left(x_{1}, \ldots, x_{n}\right)$ denote the field of rational functions over $x_{1}, \ldots, x_{n}$. We recall that a rational function has the form $f=\frac{p}{q}$ where $p, q \in \mathbb{R}\left[x_{1}, \ldots, x_{n}\right]$ are polynomials and $q \not \equiv$ 0 . Furthermore, by writing $f=\frac{p}{q}$, we assume that $p, q$ have no factors in common.

Throughout, this paper, we will investigate ODEs of the form

$$
\frac{d \vec{x}}{d t}=f(\vec{x})
$$

where $f(\vec{x}) \in \mathbb{R}[\vec{x}]$ is a polynomial.

Definition 1: The Lie derivative of a differentiable function $g: \mathbf{R}^{n} \rightarrow \mathbf{R}$ along vector field $f: \mathbf{R}^{n} \rightarrow \mathbf{R}^{n}$ is:

$$
\mathcal{L}_{f}(g)(x)=\left\langle\left(\begin{array}{c}
f_{1}(x) \\
\ldots \\
f_{n}(x)
\end{array}\right),\left(\begin{array}{c}
\frac{d g}{d x_{1}} \\
\cdots \\
\frac{d g}{d x_{n}}
\end{array}\right)\right\rangle
$$

where $\langle.,$.$\rangle is the standard inner product on \mathbf{R}^{n}$.

\section{A. Differential Variants and Lyapunov Functions}

The safety verification problem for a system seeks to prove that the system starting from a set $\vec{x} \in X_{0}$ cannot reach an unsafe set $U$. Differential variants represent an approach to establish such reachability properties [13].

Definition 2 (Differential Variant): A differential variant for an ODE $\frac{d \vec{x}}{d t}=f(x)$ over a domain $X$ is a differentiable function $g(\vec{x})$ such that for all $\vec{x} \in X, \mathcal{L}_{f}(g)>0$.

If the domain $X$ is not explicitly stated for a differential variant, we assume it is $X=\mathbb{R}^{n}$, the entire state space. The following theorem shows how differential variants are used to solve the safety verification problem:

Theorem 1: Let $g(\vec{x})$ be a differential variant for an ODE $\frac{d \vec{x}}{d t}=f(\vec{x})$ over a state space $X$, such that

1) $\forall \vec{x} \in X_{0}, g(\vec{x}) \geq 0$,

2) $\forall \vec{x} \in U, g(\vec{x})<0$

We conclude that the set $U$ is unreachable from $X_{0}$. In other words, for each time trajectory $\vec{x}(t)$ such that $\vec{x}(0) \in$ $X_{0}$, we have $\vec{x}(t) \notin U$ for all $t \geq 0$.

Just as differential variants prove unreachability properties, Lyapunov functions represent a standard approach for proving stability of ODEs. Let $\frac{d \vec{x}}{d t}=f(x)$ be an ODE. A system is Lyapunov stable over an open region $U$ around the equilibrium $\vec{x}^{*}$, if for every neighbourhood $N \subseteq U$ of $\vec{x}^{*}$ there is a (non-empty) neighbourhood $M \subset N$ such that $(\forall \vec{x}(0) \in M)(\forall t \geq 0) \vec{x}(t) \in N$. A system is asymptotically stable if it is Lyapunov stable and all trajectories starting from $U$ approach $\vec{x}^{*}$ as $t \rightarrow \infty$. Lyapunov functions are useful in proving that a system is stable in a region around the equilibrium. Without loss of generality, we assume that $\vec{x}^{*}=\overrightarrow{0}$. The definitions below are based on the terminology used in the standard text by Meiss [15].

Definition 3: A continuous and differentiable function $V(\vec{x})$ is a weak Lyapunov function over a region $U \subseteq \mathcal{X}$ iff the following conditions hold:

1) $V(\vec{x})$ is positive definite over $U$, i.e, $V(\vec{x})>0$ for all $\vec{x} \in U \backslash\{\overrightarrow{0}\}$ and $V(\overrightarrow{0})=0$.

2) $\mathcal{L}_{f}(V) \leq 0$ for all $\vec{x} \in U$.

Additionally, $V$ is a strong Lyapunov function if $\left(-\mathcal{L}_{f}(V)\right)$ is positive definite.

Weak Lyapunov functions are used to prove that a system is Lyapunov stable in a region $U$, whereas a strong Lyapunov function proves asymptotic stability. The approaches presented in this paper can be used to search for weak as well as strong Lyapunov functions.

Note 1: Throughout this paper, we will focus on approaches to compute differential variants and Lyapunov functions. The commonality between both definitions is the requirement that the Lie derivative be strictly positive in the former case, and negative semidefinite in the latter. We will first focus on the important problem of discovering functions whose derivatives are positive. The generation of Lyapunov functions is considered in Section $\mathrm{V}$.

\section{B. Darboux polynomials}

Darboux polynomials are instrumental in our search for good candidates for differential variants and Lyapunov func- 
tions, for polynomial ODEs.

Definition 4: A polynomial $p \in \mathbb{R}[\vec{x}]$ is called a Darboux polynomial (eigenpolynomial, or a polynomial second integral) iff

$$
\mathcal{L}_{f}(p)=c p
$$

where $c \in \mathbb{R}[\vec{x}]$ is a polynomial called the cofactor. When $c \equiv 0, p$ is also known as a first integral, otherwise it is called a proper Darboux polynomial.

If $p_{1}, p_{2}$ are Darboux polynomials with cofactors $c_{1}, c_{2}$, respectively, then $p_{1} p_{2}$ is also a Darboux polynomial with cofactor $c_{1}+c_{2}$. Likewise, if $p_{1}, p_{2}$ are Darboux polynomials for the same co-factor $c$ then $p_{1}+p_{2}$ is a Darboux polynomial. In general, however, the sum of two Darboux polynomials can fail to be a Darboux polynomial.

Darboux polynomials have been widely studied as a basis of algorithmic procedures for integrating ODEs [1], and deriving first integrals of ODEs [16]. Jouanolou [17] constructs a polynomial vector field for which no Darboux polynomial exist; this work has been extended later by Ollagnier and Nowicki [18]. It is also well-known that there is no apriori bound on the degree of the Darboux polynomials in terms of the degree of the RHS of the ODEs.

Example 1: Consider, first, the polynomial system due to Hahn [7]:

$$
\left\{\begin{array}{l}
\dot{x}=-x+2 x^{2} y \\
\dot{y}=-y
\end{array}\right.
$$

The polynomials $x, y, x y-1$ and their products are all examples of Darboux polynomials.

Consider the example below suggested by Parillo, as an example of a polynomial ODE without any polynomial Lyapunov functions [19]:

$$
\left\{\begin{array}{l}
\dot{x}=-x+x y \\
\dot{y}=-y
\end{array}\right.
$$

Note that $x, y$ and their products are Darboux polynomials.

\section{Finding Darboux Polynomials}

The algorithmic determination of Darboux polynomials has received some attention in the past. Darboux polynomials allow us to find rational and even algebraic first integrals, in some cases. For 2D systems, they can be used to infer general elementary first integrals ${ }^{1}$, the corresponding algorithm is known as the Prelle-Singer procedure [16]. More recently [20], efficient procedures have been described for determining rational first integrals, for planar (i.e. 2D) systems. These procedures are linked with Theorem 2.

There exist two main implementations used in the PrelleSinger procedure to discover Darboux polynomials [21]: PSODE1 and PSODE2 in the REDUCE computer algebra system. PSODE1 relies entirely on a Gröbner basis computation in the "undetermined coefficients" method for determining Darboux polynomials, whereas PSODE2 starts with a form of multivariate division. This method was later

\footnotetext{
${ }^{1}$ I.e. functions $g$, towers of logarithmic and exponential extensions to rational fractions [2], such that $\mathcal{L}_{f}(g)=0$, hence are invariant over all solutions of the ODE (1).
}

improved in [22], with less coefficients (and thus equations) to be considered. These methods require running time that is exponential in the size of the ODE, in the worst case. A recent approach based on the "ecstatic curve" was proposed by Chèze [23] reduces the problem of finding Darboux polynomials to factoring a large polynomial that is expressed as a determinant of polynomials. This approach has been shown to be polynomial time provided the coefficients of the ODE and the successive Lie derivatives belong to the field $\mathbf{Z}$ that admits efficient factorization.

Since the determination of Darboux polynomials is at the heart of the techniques suggested in this paper, we have directly implemented the ecstatic curve approach by Cheze using the computer algebra primitives available in Mathematica, and our own approach for finding Darboux polynomials that solves a class of separable bilinear equations over the coefficients of the Darboux polynomials and the co-factors. This method has been employed successfully on all our numerical examples to find Darboux polynomials. Since the actual derivation of the polynomials is orthogonal to the techniques described here, we will relegate a detailed discussion of the techniques employed in our implementation for generating Darboux polynomials to an extended version of this paper.

\section{Rational Functions And their Algebraic EXTENSIONS}

In this section, for a given domain $\mathcal{D}$, we wish to find a rational function differential variant $g \in \mathbf{R}(\vec{x})$, such that

$$
\forall x \in \mathcal{D}, \mathcal{L}_{f}(g)(x)=\tau(x)
$$

where $\tau$ a positive function on $\mathcal{D}$. We will call $\tau$ the differential remainder of $g$.

Let $g=\frac{p}{q}$ where $p, q \in \mathbb{R}[\vec{x}]$. It follows that $\mathcal{L}_{f}(g)=$ $\frac{q \mathcal{L}_{f}(p)-p \mathcal{L}_{f}(q)}{q^{2}}$. Therefore, finding a differential variant $g$ requires us to enforce that $\alpha: q \mathcal{L}_{f}(p)-p \mathcal{L}_{f}(q)$ is positive. We will use sum-of-squares programming to achieve this by expressing $\alpha=c_{0}+\tau$ for a positive semidefinite (psd) polynomial $\tau$ and $c_{0}>0$.

Similarly, finding a Lyapunov function of the form $\frac{p}{q}$ requires us to enforce, without loss of generality, that $\frac{q}{p}$ is positive definite, $\mathrm{q}$ is a strictly positive function, and $q \mathcal{L}_{f}(p)-p \mathcal{L}_{f}(q)$ is negative semi-definite. Normally, sumof-squares (SOS) relaxation applies for encoding that a given parametric polynomial form $p(\vec{c}, \vec{x})$ with unknown parameters is SOS. However, the reader will notice that enforcing the positive semi-definiteness of $q \mathcal{L}_{f}(p)-p \mathcal{L}_{f}(q)$ reduces to solving bilinear matrix inequalities (BMIs) rather than LMIs. Furthermore, it is well known that solving BMIs is computationally harder than solving LMIs. Therefore, in this section, we relate the existence of a restricted class of rational function differential variants to that of Darboux polynomials.

We first note the following result:

Lemma 1: Let $g=\frac{p}{q}$ be a rational function such that (a) $q$ is a Darboux polynomial with co-factor $c$, and (b) $\alpha q$ is a positive polynomial where $\alpha=\mathcal{L}_{f}(p)-c p$. 
It follows that $g$ is a rational differential variant.

Proof: Note that $\mathcal{L}_{f}(g)=\frac{q \alpha}{q^{2}}$ is positive.

Note 2: As a side condition, we note that using a rational function as a differential variant or a Lyapunov function is possible only over a domain $\mathcal{D}$ where the function is well defined. Often, we seek rational functions $\frac{p}{c+q}$ where $c>0$ is a constant, and $p, q$ are homogeneous psd polynomials.

Conversely, we show that if $r=\frac{p}{q}$ is a rational differential variant such that $\mathcal{L}_{f}(r)$ is a polynomial, then $q$ must be a Darboux polynomial and the conditions in Lemma 1 automatically hold.

Theorem 2: Let $r=\frac{p}{q}$ be a rational function that is a differential variant, where $p, q$ are relatively prime. We assume that $\mathcal{L}_{f}(r)$ is a polynomial. It follows that

- $q$ is a Darboux polynomial with cofactor $c$, i.e, $\mathcal{L}_{f}(q)=$ $c q$

- and $\alpha q$ is positive, where $\alpha=\mathcal{L}_{f}(p)-c p$.

Proof: Suppose $r=\frac{p}{q}$ is a differential variant, with $p$ and $q$ relatively prime. Given that $f$ is a polynomial vector field, $\mathcal{L}_{f}(r)$ is a positive rational function $\gamma=\frac{\alpha}{q}$ for some polynomial $\alpha$. We have $\mathcal{L}_{f}(r)=\frac{\mathcal{L}_{f}(p) q-p \mathcal{L}_{f}(q)}{q^{2}}=\gamma$. Hence,

$$
\begin{aligned}
\mathcal{L}_{f}(p) q-p \mathcal{L}_{f}(q) & =\gamma q^{2} \\
& =\alpha q
\end{aligned}
$$

Since $\alpha$ is a polynomial, this implies that $q$ divides $p \mathcal{L}_{f}(q)$. Therefore, since $p$ and $q$ are relatively prime, $q$ divides $\mathcal{L}_{f}(q)$. Thus, $q$ is a Darboux polynomial. Let $c$ be its cofactor, we then have $\mathcal{L}_{f}(p)-c p=\alpha$.

Finally, asking for $\gamma$ to be positive means that $\alpha q$ is itself positive.

\section{A. Algebraic Extensions to Rational Variants}

We prove in this section that if we have a differential variant which is algebraic on rational functions with a rational differential remainder, then we know there exists a rational differential variant. The notion of algebraic extension to rational function encompasses functions such as $\sqrt{\frac{p}{q}},\left(\frac{p}{q}\right)^{\frac{1}{3}}$ that are expressible as algebraic equations involving rational functions. The main result is that if such differential variants can be found that have a rational differential remainder, then we can also find rational differential variants.

The following theorem is based on well known class reduction theorems for first integrals [1].

Theorem 3: Let $c$ be a non-trivial algebraic function over $\mathbf{R}\left(x_{1}, \ldots, x_{n}\right)$. If $c$ is a differential variant for ODE (1), with a rational differential remainder, then there exists a non trivial rational function $\hat{c} \in \mathbf{R}\left(x_{1}, \ldots, x_{n}\right)$, which is also a differential variant for ODE (1).

Proof: Let $c$ be an algebraic function over $R\left(x_{1}, \ldots, x_{n}\right)$ which is a differential variant for the polynomial vector field $f$, so that $\mathcal{L}_{f}(c)=\tau$ with $\tau$ rational and positive. The fact that $c$ is algebraic means that there exists a polynomial $p$ such that $p(c)$ is identically zero:

$$
p(c): q_{0}+q_{1} c+\ldots+q_{s-1} c^{s-1}+c^{s}=0 .
$$

Here, $q_{i}, i=0, \ldots, s-1$ are rational functions over $\mathbb{R}\left(x_{1}, \ldots, x_{n}\right)$. Furthermore, without loss of generality, we choose $p$ to be a polynomial of minimal degree, and let the coefficient of its leading term $c^{s}$ be identical to 1 . In other words, no other non-zero polynomial $\hat{q}$ of degree strictly less than $s$ can satisfy $\hat{q}(c)=0$.

Applying Lie derivative to both sides of Equation 2, $f$ that:

$$
\begin{aligned}
& \mathcal{L}_{f}\left(q_{0}\right)+\mathcal{L}_{f}\left(q_{1}\right) c+\ldots+\mathcal{L}_{f}\left(q_{s-1}\right) c^{s-1} \\
& +\tau\left(q_{1}+2 q_{2} c+\ldots+(s-1) q_{s-1} c^{s-2}+s c^{s-1}\right)=0
\end{aligned}
$$

I.e. regrouping terms:

$$
\hat{p}(c):\left(\begin{array}{c}
\left.\mathcal{L}_{f}\left(q_{0}\right)+\tau q_{1}\right)+\left(\mathcal{L}_{f}\left(q_{1}\right)+2 \tau q_{2}\right) c \\
+\ldots+ \\
\left(\mathcal{L}_{f}\left(q_{s-1}\right)+s \tau\right) c^{s-1}
\end{array}\right)=0
$$

$\hat{p}$ has a lower degree than $p$ and $\hat{p}(c)=0$. This directly contradicts the fact that $p$ is the minimal polynomial for $c$, unless the coefficients of $\hat{p}$ vanish:

$$
\begin{aligned}
& \mathcal{L}_{f}\left(q_{0}\right)+\tau q_{1}=0 \\
& \mathcal{L}_{f}\left(q_{1}\right)+2 \tau q_{2}=0 \\
& \ldots \\
& \mathcal{L}_{f}\left(q_{s-1}\right)+s \tau=0
\end{aligned}
$$

The last equation shows that $-q_{s-1}$ is a rational function such that $\mathcal{L}_{f}\left(-q_{s-1}\right)=s \tau$ is a positive rational function. This proves, in case $\tau$ is different from the zero function, that we have a non trivial rational function $-q_{s-1}$ as soon as we have an algebraic differential variant.

For the case $\tau=0$ and $q_{s-1}$ is not constant, we still have a non-trivial first integral (so, a differential variant).

Now the last case is $\tau=0$ and $q_{s-1}$ is a constant. One of the $q_{i}, i=0, \ldots, s-2$ is non trivial at least. Then we have $\mathcal{L}_{f}\left(q_{i}\right)=0$ hence $q_{i}$ is a non-trivial rational first integral and therefore a differential variant.

\section{B. Algorithmic sketch}

Following Lemma 1 and Theorem 2, we find rational differential variants, we first start by finding a set of Darboux polynomials $q_{1}, \ldots, q_{k}$ of degree up to $D$. Each $q_{i}$ is the starting point for finding a rational differential variant. Note that our approach for generating these polynomials start with an initial set of irreducible polynomials that cannot be written as the product of two Darboux polynomials, and generates new polynomials by multiplying the irreducible Darboux polynomials together.

For each Darboux polynomial $q_{i}$ with co-factor $c_{i}$, we wish to find a polynomial $p_{i}$ such that $\mathcal{L}_{f}\left(p_{i}\right)-c_{i} p_{i}=$ $\alpha_{i}$ and $\alpha_{i} q_{i}$ is a positive polynomial. We achieve this by starting with a parametric polynomial form for $1+p_{i}$, i.e, $p_{i}=\sum_{\mathbf{i} \in I} b_{\mathbf{i}} x^{\mathbf{i}}$, where $\mathbf{i}$ is a multi index, $x^{\mathbf{i}}$ stands for the corresponding monomial and $b_{\mathbf{i}}$ is an unknown parameter. We then compute $\left(\mathcal{L}_{f}(p)-c p\right) q$ as a polynomial, which has as coefficients for each monomial $x^{\mathrm{i}}$ some linear function of the $b_{\mathbf{i}}$. The conditions for which $\alpha q=\left(\mathcal{L}_{f}(p)-c p\right) q$ is a positive polynomial, are determined using the Sum of 
Squares relaxation [24]. If the resulting problem is infeasible, we consider the next Darboux polynomial in our set.

Example 2: Consider again Hahn's example (Ex. 1). We note that $q=x$ is a Darboux polynomial with cofactor $c=$ $2 x y-1$. We are looking for a polynomial $p$ such that $\mathcal{L}_{f}(p)-$ $(2 x y-1) p=\alpha$ and $\alpha x$ is a psd polynomial.

Starting from a generic polynomial of maximal total degree 2: $p=\left(b_{0}+b_{y} y+b_{y^{2}} y^{2}\right)+\left(b_{x}+b_{x y} y\right) x+b_{x^{2}} x^{2}$. We note that $\mathcal{L}_{f}(p)-(2 x y-1) p=\left(b_{0}-b_{y^{2}} y^{2}\right)+\left(\left(2 b_{0}-b_{x y}\right) y-\right.$ $\left.2 b_{y} y^{2}+2 b_{y^{2}} y^{3}\right) x+\left(-b_{x^{2}}+4 b_{x} y+4 b_{x y} y^{2}\right) x^{2}+\left(6 b_{x^{2}} y\right) x^{3}$.

A simple solution for $\alpha x$ to be SOS is given by $p=-y$. We then note that $\alpha q=2 y^{2} x^{2}$ is indeed a psd polynomial. Therefore, we conclude that $\frac{x}{y}$ is a differential variant.

Looking at all the Darboux polynomials we found for Example 1 in turn, we obtain the following rational functions differential variants:

- $q=x^{2}: p=x^{2}$ hence $r=1$,

- $q=x: p=-y$ hence $r=-\frac{y}{x}$,

- $q=x y: p=b_{x^{2}} x^{2}+b_{y^{2}} y^{2}$, hence $r=\frac{b_{x^{2}} x^{2}+b_{y^{2}} y^{2}}{x y}$,

- $q=-1+x y: p=x^{2}$, hence $r=\frac{x^{2}}{x y-1}$,

- $q=y^{2}: p=x y$, hence $r=\frac{x}{y}$,

- $q=y: p=x$, hence again, $r=\frac{x}{y}$,

- $q=1: p=-y^{2}$, hence $r=-y^{2}$.

Note that our approach finds some non trivial rational function differential variants including $\frac{x}{y}$ and $\frac{x^{2}}{x y-1}$. We discuss the derivation of Lyapunov functions using these variants in Section V.

\section{TRAnSCEndental Functions}

We now look at results that enable us to generate restricted classes of differential variants and Lyapunov functions that involve logarithmic and exponential terms. From the theory of differential fields and "integration in finite terms" (as used in e.g. computed algebra, see [2]), we know that "elementary functions" are obtained by successive elementary extensions of the differential field of rational functions, which may provide for interesting solutions.

\section{A. Logarithmic extensions}

Consider the following ODE modeling predator-prey dynamics (see [16])

$$
\begin{aligned}
\frac{d x}{d t} & =a x-b x y \\
\frac{d y}{d t} & =-c y+d x y
\end{aligned}
$$

Here, $a, b, c, d>0$ are parameters. There is no explicit solution to this ODE in "finite terms". However, using a log extension to rational functions, we obtain the first integral: $F(x, y)=d x+b y-c \log (x)-a \log (y)$. This suggests extending rational functions using logarithmic terms.

We wish search for differential variants $V$ of the form:

$$
V=\ln \left(r_{1}\right)+p_{2}
$$

where $r_{1}$ is a rational function and $p_{2}$ is a polynomial. We further restrict our search for the case where the differential remainder is a polynomial. Trying to solve more general cases requires again having to solve expensive polynomial matrix inequalities (PMIs). Let $r_{1}=\frac{p_{1}}{q_{1}}$ where $p_{1}, q_{1}$ are relatively prime.

Theorem 4: $V=\log \left(\frac{p_{1}}{q_{1}}\right)+p_{2}$ is a differential variant with a polynomial differential remainder if and only if:

- $p_{1}$ is a Darboux polynomial (with cofactor $\beta$ )

- $q_{1}$ is a Darboux polynomial (with cofactor $\alpha$ )

- $\mathcal{L}_{f}\left(p_{2}\right)=\alpha-\beta+\tau$, where $\tau$ is a positive polynomial.

Proof: Writing $r_{1}=\frac{p_{1}}{q_{1}}$, the Lie derivative of $V$ along vector field $f$ is:

$$
\begin{aligned}
\mathcal{L}_{f}(V) & =\frac{\mathcal{L}_{f}\left(p_{1}\right)}{p_{1}}-\frac{\mathcal{L}_{f}\left(q_{1}\right)}{q_{1}}+\mathcal{L}_{f}\left(p_{2}\right) \\
& =\beta-\alpha+\alpha-\beta+\tau \\
& =\tau
\end{aligned}
$$

which is a positive polynomial.

Conversely, let $\mathcal{L}_{f}(V)=p$, be a positive polynomial. Therefore, we have:

$$
\frac{\mathcal{L}_{f}\left(p_{1}\right)}{p_{1}}-\frac{\mathcal{L}_{f}\left(q_{1}\right)}{q_{1}}+\mathcal{L}_{f}\left(p_{2}\right)=p
$$

As $p_{1}$ and $q_{1}$ are relatively prime, and $p$ and $p_{2}$ are polynomials, it follows that $p_{1} \mid \mathcal{L}_{f}\left(p_{1}\right)$ and $q_{1} \mid \mathcal{L}_{f}\left(q_{1}\right)$. I.e, $p_{1}, q_{1}$ are Darboux polynomial. Finally, the differential remainder $p$ is identified with the positive polynomial $\tau$ in the statement of the theorem.

\section{B. Algorithmic sketch}

Once again, we assume that we have found Darboux polynomials $q_{1}, \ldots, q_{N}$. We examine two relatively prime Darboux polynomials $q_{1}, q_{2}$ with co-factors $c_{1}, c_{2}$, respectively, and attempt to find a polynomial $p$ such that $\mathcal{L}_{f}(p)-$ $c_{1}+c_{2}$ is a positive polynomial. This is performed by fixing a parametric polynomial for $p$ with unknown coefficients and using SOS programming to discover if a solution exists. If so, the solution yields a logarithmic differential variant involving $\log \left(\frac{q_{1}}{q_{2}}\right)$. Note that the domain of existence of this differential variant is given by $q_{1}>0 \wedge q_{2}>0$.

Example 3: Consider once again the system from Example 1. The non trivial Darboux polynomials of degree one are $x$ and $y$, with co-factors $\beta=y-1$ and $\alpha=-1$, respectively. Hence we consider differential variants of the form $V=\log \left(\frac{x}{y}\right)+p_{2}$. Applying Theorem 4, we obtain the condition for $p_{2}$ as

$$
\mathcal{L}_{f}\left(p_{2}\right)+(y-1)-1=\tau \text { is a positive polynomial. }
$$

Setting $p_{2}=\alpha_{x} x+\alpha_{y} y$, for unknown parameters $\alpha_{x}, \alpha_{y}$, we compute:

$$
\mathcal{L}_{f}\left(p_{2}\right)=-\alpha_{y} y+\alpha_{x}(y-1) x
$$

Therefore, $\tau=\mathcal{L}_{f}\left(p_{2}\right)+y=\left(1-\alpha_{y}\right) y+\alpha_{x}(y-1) x$. We find that $\alpha_{y}=1, \alpha_{x}=0$, yields $\tau=0$. Therefore, we have discovered a differential variant: $V(x, y)=\log \left(\frac{x}{y}\right)+y$ for this system. This is defined whenever $x>0, y>0$. 


\section{Exponential extensions}

The case of exponential extensions is, in general, more involved than logarithmic extensions of Section IV-A. The idea is to find rational fraction $r_{1} \in \mathbf{R}\left(x_{1}, \ldots, x_{n}, z_{1}, \ldots, z_{m}\right)$ involving extra auxiliary variables $z_{1}, \ldots, z_{m}$ and polynomials $p_{1}, \ldots, p_{m} \in$ $\mathbb{R}\left[x_{1}, \ldots, x_{n}\right]$ such that $r_{1}\left(x, e^{p_{1}(x)}, \ldots, e^{p_{m}(x)}\right)$ is a differential variant. To do so, we will simply fix the set of polynomial terms $p_{1}, \ldots, p_{m}$ a priori. A common choice is to find monomials up to a given degree. We will then extend our original system of ODEs by adding extra auxiliary variables $z_{1}, \ldots, z_{m}$, wherein $z_{i}$ represents $\exp \left(p_{i}(\vec{x})\right)$. Therefore, we set $\frac{d z_{i}}{d t}=\mathcal{L}_{f}\left(p_{i}\right) z_{i}$. Since $p_{1}, \ldots, p_{m}$ are polynomials, the extended system over $x_{1}, \ldots, x_{n}, z_{1}, \ldots, z_{m}$ is a polynomial ODE over which the techniques for finding rational and logarithmic invariants can be applied after searching for Darboux polynomials.

Lemma 2: Let $p \in \mathbb{R}\left[x_{1}, \ldots, x_{n}\right]$ and $r_{1} \in$ $\mathbf{R}\left(x_{1}, \ldots, x_{n}, z\right)$. The function $r_{1}\left(x, e^{p(x)}\right)$ is a differential variant of the polynomial ODE (1) if and only if $r_{1}$ is a rational differential variant of the following auxiliary ODE: $\left(x \in \mathbf{R}^{n}\right)$ :

$$
\begin{aligned}
\dot{x} & =f(x) \\
\dot{z} & =\mathcal{L}_{f}(p) u
\end{aligned}
$$

\section{Determining Lyapunov Functions}

Recall that Lyapunov functions are $C^{1}$ functions $V: \mathcal{D} \rightarrow$ $\mathbf{R}$ over a region of stability $\mathcal{D} \subseteq \mathbf{R}^{n}$, such that $\mathcal{L}_{f}(V) \leq 0$ and $V$ is positive definite over $\mathcal{D}$. Without loss of generality, we assume that $\overrightarrow{0} \in \mathcal{D}$ is the sole equilibrium, whose stability is to be established.

In particular, global Lyapunov functions $V$ are such that $-V$ are differential variants in the sense of Definition 2, that we helped determine in Sections III-B in the rational case and IV-B in the logarithmic case.

Local Lyapunov functions are also such that $-V$ are "locally" differential variants, that can also be determined as in Sections III-B and IV-B. For a given $\mathcal{D}$ described by a set of polynomial inequality constraints, SOS programming is used in conjunction with Putinar's positivstellensatz to prove that a given polynomial is positive semidefinite over $\mathcal{D}$.

The key challenge therefore lies in reusing the techniques described thus far, which have focused on deriving differential variants to finding Lyapunov function. Specifically, we wish to enforce that the function $V$ is positive definite while at the same time enforcing the negative semi-definiteness of its Lie derivative.

\section{A. Combining Rational Differential Variants}

We describe a trick, that given $k$ rational differential variants $r_{1}, \ldots, r_{k} \in \mathbf{R}\left(x_{1}, \ldots, x_{n}\right)$, combines them to yield a Lyapunov function. We choose a polynomial $P\left(z_{1}, \ldots, z_{k}\right)$ and assume that a polynomial combination $V(\vec{x})=P\left(r_{1}(\vec{x}), \ldots, r_{k}(\vec{x})\right)$ yields the required Lyapunov function over the given domain $\mathcal{D}$ containing the equilibrium point. Since $P$ is unknown, we parameterize $P$ in the standard way and compute constraints on the unknown parameter. The requirements over $P$ are as follows:

1) $P\left(r_{1}(\overrightarrow{0}), \ldots, r_{k}(\overrightarrow{0})\right)=0$.

2) Forall $\vec{x} \in \mathcal{D} \backslash\{\overrightarrow{0}\}$, we require $P\left(r_{1}, \ldots, r_{k}\right)>0$.

3) Finally, we require $P\left(z_{1}, \ldots, z_{k}\right)$ is non-increasing over $\mathcal{D}$. I.e, for all $1 \leq j \leq k$, we require $\frac{\partial P\left(z_{1}, \ldots, z_{k}\right)}{\partial z_{j}}$ is negative semi-definite.

Since each $r_{i}=\frac{p_{i}}{q_{i}}$ is assumed to be well defined over $\mathcal{D}$, we assume without loss of generality that $q_{i}>0$ over $\mathcal{D}$.

Let $P=P\left(\vec{c}, z_{1}, \ldots, z_{k}\right)$ be the parametric form for the polynomial $P$. Condition 1 yields affine constraints over $\vec{c}$. Next, we can use SOS programming to enforce condition 2. Here, we can replace $r_{i}$ with the polynomial $p_{i} \Pi_{j \neq i} q_{j}$. Note that $P$ is often taken to be an affine form in our example, and therefore this does not cause a large blowup in the degree of the resulting polynomial. Finally, condition 3 is also encoded using SOS programming. As a result, we obtain an LMI that can be solved to obtain coefficients for the polynomial form $P$. Using $P$, we obtain a Lyapunov function $V(\vec{x})$.

Example 4 (Hahn): Returning to Example 2, we recall the differential variants $g_{1}=-y^{2}, g_{2}=\frac{x}{y}$ and $g_{3}=\frac{x^{2}}{x y-1}$ discovered over the domain $x y<1$. We find that the polynomial $P\left(z_{1}, z_{2}, z_{3}\right)=\frac{-z_{1}}{2}+\frac{-z_{3}}{2}$. Setting, $V=P\left(g_{1}, g_{2}, g_{3}\right)$, yields the function $V(x, y)=\frac{y^{2}}{2}+\frac{x^{2}}{2(1-x y)}$, which is indeed a Lyapunov function over the domain $\mathcal{D}=\left\{(x, y) \in \mathbf{R}^{2} \mid\right.$ $x y<1\}$. In previous work by Zubov, Davison, Vannelli and Vidyasagar [25], this function was also originally found by Zubov's method [7].

\section{B. Logarithmic Lyapunov functions}

Similarly as in Section V-A, we have to ensure that the differential variants $F=\log \left(r_{1}\right)+p_{2}$, that we determined in Section IV-B are indeed Lyapunov function. We will assume that the function $r_{1}$ is strictly positive over the domain $\mathcal{D}$.

A pragmatic method is to generate $F=\log \left(r_{1}\right)+p_{2}$ with $\mathcal{L}(F)$ positive semi-definite, and check if $V=-F$ can be a Lyapunov function. The sufficient test simply ensures that (a) $r_{1}(\vec{x})=1$ if and only if $\vec{x}=0$, (b) $r_{1}(\vec{x})<1$ over $\mathcal{D} \backslash\{\overrightarrow{0}\}$, and (c) $p_{2}(\vec{x})$ is negative definite over $\mathcal{D}$. It is easy to show that if all these conditions are satisfied, then $V=-F$ is indeed a Lyapunov function.

\section{NUMERICAL EXAMPLES}

Thus far, we have illustrated our approach to derive nonpolynomial Lyapunov functions and differential variants for two examples proposed by Hahn and Parillo (Cf. Example 1). We demonstrate our ideas on some further numerical examples. The numerical examples shown below involved our implementations for finding Darboux polynomials in MATLAB and Mathematica. We used SOSTOOLS and obtained rather interesting but complex rational and logarithmic differential invariants. Given the Darboux polynomials, it used less than 1s of execution time for all those examples. The calculations are performed here by hand or using computer algebra software Mathematica to demonstrate simpler functions that are easier to check. In theory, these can be generated by SOSTOOLS with an appropriate set of constraints and objective 
functions. Our method will be implemented as a monolithic, stand alone toolbox in MATLAB or Mathematica in the near future.

\section{A. A two prey one predator model}

In [26], the following two prey one predator Lotka-Volterra system is studied:

$$
\begin{aligned}
\dot{x}_{1} & =x_{1}\left(a_{1}-b_{1} y\right) \\
\dot{x}_{2} & =x_{2}\left(a_{2}-b_{2} y\right) \\
\dot{y} & =y\left(-c+d_{1} x_{1}+d_{2} x_{2}\right)
\end{aligned}
$$

$x_{1}, x_{2}$ and $y$ represent the biomass of populations, hence are non-negative. The domain of interest is $\mathcal{D}=$ $\left\{\left(x_{1}, x_{2}, y\right) \mid x_{1}, x_{2}, y \geq 0\right\}$. We set the parameter values to $a_{1}=b_{1}=1, a_{2}=1, b_{2}=2$ and $c=d_{1}, d_{2}=1$.

a) Darboux Polynomials: Darboux polynomials include $x_{1}, x_{2}$ and $y$ with respective cofactors $0,1-y, 1-2 y$ and $-1+x_{1}+x_{2}$. Higher degree Darboux polynomials are obtained as products of these Darboux polynomials. As a consequence, $x_{1} y$ is Darboux with cofactor $-x_{1}+x_{2}-y$.

b) Logarithmic Variants: We use now the algorithm of Section IV-B to determine polynomials $p_{1}, p_{2}$ and $q_{1}$ such that $V=\log \left(\frac{p_{1}}{q_{1}}\right)+p_{2}$ is a differential variant. Note that we require $p_{1}, q_{1}$ to be Darboux polynomials.

We select $p_{1}=x_{1} y$ (with cofactor $\beta=-x_{1}+x_{2}-y$ ) and $q_{1}=1$ (with cofactor $\alpha=0$ ). We search for $p_{2}=\alpha_{0}+$ $\alpha_{x_{1}} x_{1}+\alpha_{x_{2}} x_{2}+\alpha_{y} y$, as an unknown, generic polynomial of degree one.

We now have to determine the coefficients of $p_{2}$ so that $\tau=\beta-\alpha+\mathcal{L}_{f}\left(p_{2}\right)$ is a psd polynomial over $\mathcal{D}$. Setting up this condition and solving yields the polynomial $p_{2}=$ $-x_{1}-\frac{x_{2}}{2}-y$. The resulting polynomial $\tau$ is then $\frac{x_{2}}{2}$ which is positive on $\mathcal{D}$.

As a result, we discover the differential variant

$$
V=\log \left(x_{1} y\right)-x_{1}-\frac{x_{2}}{2}-y \text {. }
$$

We compare our result to the differential variant $V_{1}$ noted by [26], for the more general case $\frac{a_{1}}{b_{1}}>\frac{a_{2}}{b_{2}}$ :

$$
V_{1}(x, y)=\frac{d_{1} x_{1}}{b_{1}}+\frac{d_{2} x_{2}}{b_{2}}+y-\frac{c}{b_{1}} \ln x_{1}-\frac{a_{1}}{b_{1}} \ln y
$$

c) Rational Variants: Looking for rational differential variants using algorithm III-B, we choose the Darboux polynomial $q=x_{1}^{2}$, with cofactor $c=2(1-y)$.

We search for a numerator $p$ of degree 1 parameterized by the template: $p=\alpha_{0}+\alpha_{x_{1}} x_{1}+\alpha_{x_{2}} x_{2}+\alpha_{y} y$. We wish to find a $p$ such that $q\left(\mathcal{L}_{f}(p)-c p\right)$ is a psd polynomial over the domain $\mathcal{D}=\left\{\left(x_{1}, x_{2}, y\right) \mid x_{1}, x_{2}, y \geq 0\right\}$. Solving, we obtain $\alpha_{x_{2}}=-1$, and $\alpha_{0}=\alpha_{x_{1}}=0$. This yields the polynomial $p=-x_{2}$. Therefore, our approach results in a differential variant $\frac{p}{q}=\frac{-x_{2}}{x_{1}^{2}}$.

In fact, our approach rather simply discovers an instance of the Lyapunov function stated in [26], $V_{2}(x, y)=\frac{x_{2}^{b_{1}}}{x_{1} b_{2}}$ for the more general case.
B. The model of May-Leonard: a 3-species competitive Lotka-Volterra system

We now focus on an example of an ODE involving parameters. In [27], the authors consider the following system:

$$
\begin{aligned}
& \dot{x}_{1}=x_{1}\left(1-x_{1}-\alpha x_{2}-\beta x_{3}\right) \\
& \dot{x}_{2}=x_{2}\left(1-\beta x_{1}-x_{2}-\alpha x_{3}\right) \\
& \dot{x}_{3}=x_{3}\left(1-\alpha x_{1}-\beta x_{2}-x_{3}\right)
\end{aligned}
$$

where $\alpha, \beta$ are non negative.

For this system, which is more general than the one of Section VI-A, we treat the two parameters $\alpha$ and $\beta$ as state variables, letting $\dot{\alpha}=0$ and $\dot{\beta}=0$. We now have to consider polynomials on $x_{1}, x_{2}, x_{3}$ and $\alpha, \beta$.

d) Darboux Polynomials: $\alpha, \beta, x_{1}, x_{2}$ and $x_{3}$ are trivially the Darboux polynomials of total degree less than or equal to one, with respective cofactors $0,0,1-x_{1}-\alpha x_{2}-$ $\beta x_{3}, 1-\beta x_{1}-x_{2}-\alpha x_{3}$ and $1-\alpha x_{1}-\beta x_{2}-x_{3}$.

When $\alpha+\beta=2$, we discover the Darboux polynomial $x_{1}+x_{2}+x_{3}$ with cofactor $1-x_{1}-x_{2}-x_{3}$.

e) Rational Variants: We first look for rational differential variants, using the algorithm of Section III-B, when $\alpha+\beta=2$. We use the Darboux polynomial $q=\left(x_{1}+x_{2}+\right.$ $\left.x_{3}\right)^{3}$ with cofactor $c=3\left(1-x_{1}-x_{2}-x_{3}\right)$. Our goal is to search for a polynomial $p$ such that $q(\underbrace{p-c \mathcal{L}_{f}(p)}_{\alpha})$ is a psd polynomial. We parameterize $p$ as a degree 3 polynomial, which is too large to be shown here.

Our search yields the polynomial $p=x_{1} x_{2} x_{3}$ and the function $V\left(x_{1}, x_{2}, x_{3}\right)=\frac{x_{1} x_{2} x_{3}}{\left(x_{1}+x_{2}+x_{3}\right)^{3}}$. In fact, we find that $\mathcal{L}_{f}(V) \equiv 0$, and therefore $V$ is a first integral of this LotkaVolterra system, when $\alpha+\beta=2$.

When $\alpha+\beta \neq 2$, the authors of [27] find that a small modification (by a constant factor) of the rational function above, $V\left(x_{1}, x_{2}, x_{3}\right)=(\alpha+\beta-2) \frac{x_{1} x_{2} x_{3}}{\left(x_{1}+x_{2}+x_{3}\right)^{3}}$ is a Lyapunov function. Our method fails to find this Lyapunov function in for this case (although we find a first integral when $\alpha+\beta=2)$. This is because $\alpha+\beta \neq 2,\left(x_{1}+x_{2}+x_{3}\right)^{3}$ is not Darboux. Stated differently, the differential remainder of $V$ is of the form $\frac{\alpha}{\left(x_{1}+x_{2}+x_{3}\right)^{3}}$ (but of a more general form $\frac{\alpha}{\left(x_{1}+x_{2}+x_{3}\right)^{4}}$, see the restriction in Theorem 2). This is a limitation of our approach.

f) Logarithmic Variants: We now search for logarithmic differential variants, using the algorithm of Section IV-B, for the case $\alpha+\beta=2$.

We use the Darboux polynomial $q_{1}=x_{1} x_{2} x_{3}$ with cofactor $c_{1}=3\left(1-x_{1}-x_{2}-x_{3}\right)$, the algorithm will have to choose among the Darboux polynomials for $p_{1}$. We choose $p_{1}=1$, with cofactor $c_{2}=0$. Now we are looking for $p_{2}$ such that $\mathcal{L}_{f}\left(p_{2}\right)+c_{2}-c_{1}=\tau$, psd polynomial. We parameterize $p_{2}$ as an affine expression and discover the polynomial $3\left(x_{1}+x_{2}+x_{3}\right)$. Therefore, we find a logarithmic Lyapunov function [27]: $V\left(x_{1}, x_{2}, x_{3}\right)=$ $3\left(x_{1}+x_{2}+x_{3}\right)-\log \left(x_{1} x_{2} x_{3}\right)$, which is only minimum on $\mathcal{D}=\left\{\left(x_{1}, x_{2}, y\right) \mid x_{1}, x_{2}, y \geq 0\right\}$ when $x_{1}, x_{2}$ and $y$ are all zero, proving the stability of equilibrium point $(0,0,0)$. 
TABLE I

COMPUTATION TIMES FOR FINDING DARBOUX POLYNOMIALS. THE COLUMN FOUND/KNOWN CONTRASTS THE NUMBER FOUND AUTOMATICALLY AGAINST THOSE FOUND BY HAND CALCULATIONS.

\begin{tabular}{|l|l|l|}
\hline Example & Time & Found/Known \\
\hline Hahn (Ex. 1) & $15 \mathrm{~s}$ & $2 / 3$ \\
Parillo (Ex. 1) & $19 \mathrm{~s}$ & $2 / 2$ \\
Lotka-Volterra (Sect. VI-A) & $98 \mathrm{~s}$ & $3 / 3$ \\
May-Leonard (Sect. VI-B) & $2351 \mathrm{~s}$ & $5 / 5$ \\
May-Leonard, $\alpha+\beta=2$ & $451 \mathrm{~s}$ & $5 / 6$ \\
\hline
\end{tabular}

Our method discovers another previously unknown logarithmic Lyapunov function for this example. We choose the Darboux polynomials $p_{1}=x_{1}+x_{2}+x_{3}$ with cofactor $1-x_{1}-x_{2}-x_{3}$ and $q_{1}=1$ with cofactor 0 . Looking for total degree one polynomials $p_{2}$, we find a simpler Lyapunov function $V_{1}\left(x_{1}, x_{2}, x_{3}\right)=x_{1}+x_{2}+x_{3}-\log \left(x_{1}+x_{2}+x_{3}\right)$.

\section{Computation Times}

The total time required can be decomposed into the time for finding Darboux polynomials and the time required for SOS programming. The latter component was always under a second for most examples. Table I reports the time taken for finding Darboux polynomials for the various benchmarks presented in this section. In each case, we search for Darboux polynomials of degree less than 3 and with cofactor of degree less than 3, we get the following results:

\section{CONCLUSION}

To conclude, we note that Darboux polynomials are useful tools for the discovery of non-polynomial (rational, logarithmic and transcendental) differential variants and Lyapunov functions. Our future work will focus on finding nonpolynomial Lyapunov functions that are formal power series, or use piecewise polynomials. Extensions to hybrid dynamical systems combining discrete and continuous dynamics will constitute an important future direction.

Acknowledgments The authors would like to thank the anonymous reviewers for their careful reading and insightful comments. This work was supported by the ANR-12-INSE0007-02 CAFEIN project and the US National Science Foundation (NSF) under CAREER Award \# 0953941. All opinions expressed here are those of the author and not necessarily those of the NSF.

\section{REFERENCES}

[1] A. Goriely, Integrability and Nonintegrability of Dynamical Systems, ser. Advanced Series in Nonlinear Dynamics. World Scientific, 2001. [Online]. Available: http://books.google.co.uk/books?id=HtbsBIbGlycC

[2] R. Risch, The Problem of Integration in Finite Terms. University of California, 1968.

[3] S. Boyd, L. El Ghaoui, E. Feron, and V. Balakrishnan, Linear Matrix Inequalities in System and Control Theory, ser. Studies in Applied Mathematics. Philadelphia, PA: SIAM, June 1994, vol. 15.
[4] B. Tibken, "Estimation of the domain of attraction for polynomial systems via LMIs," in IEEE CDC, vol. 4. IEEE Press, 2000, pp. 3860-3864 vol.4.

[5] D. Henrion and J. B. Lasserre, "Inner approximations for polynomial matrix inequalities and robust stability regions," IEEE Transactions on Automatic Control, vol. 57, no. 6, pp. 1456-1467, 2012.

[6] D. Henrion and M. Korda, "Convex computation of the region of attraction of polynomial control systems," 2013.

[7] V. Zubov and A. Liapunov, Methods of A. M. Lyapunov and Their Application, ser. Translation series. U.S. Atomic Energy Commission, 1961.

[8] A. Vannelli and M. Vidyasagar, "Maximal lyapunov functions and domains of attraction for autonomous nonlinear systems," Automatica, vol. 21, no. 1, pp. 69-80, 1985.

[9] P. A. Parillo, "Semidefinite programming relaxation for semialgebraic problems," Mathematical Programming Ser. B, vol. 96, no. 2, pp. 293 320, 2003.

[10] S. Prajna, A. Papachristodoulou, P. Seiler, and P. A. Parrilo, SOSTOOLS: Sum of squares optimization toolbox for MATLAB, Available from http://www.cds.caltech.edu/sostools and http://www.mit.edu/ parrilo/sostools, 2004.

[11] A. Papachristodoulou and S. Prajna, "Analysis of non-polynomial systems using the sum of squares decomposition," in Positive Polynomials in Control, ser. Lecture Notes in Control and Information Science, D. Henrion and A. Garulli, Eds., 2005, vol. 312, pp. 23-43.

[12] A. Tiwari and G. Khanna, "Non-linear systems: Approximating reach sets," in HSCC, ser. LNCS, vol. 2993. Springer, 2004, pp. 477-492.

[13] A. Platzer, "Differential dynamic logic for hybrid systems," J. Autom Reasoning, vol. 41, no. 2, pp. 143-189, 2008.

[14] S. Prajna and A. Jadbabaie, "Safety verification using barrier certificates," in HSCC, ser. LNCS, vol. 2993. Springer, 2004, pp. 477-492.

[15] J. D. Meiss, Differential Dynamical Systems. SIAM publishers, 2007.

[16] M. J. Prelle and M. F. Singer, "Elementary first integrals of differential equations," in Proceedings of the fourth ACM symposium on Symbolic and algebraic computation, ser. SYMSAC ' 81 . New York, NY, USA: ACM, 1981, pp. 30-35. [Online]. Available: http://doi.acm.org/10.1145/800206.806368

[17] J. Jouanolou, Equations de Pfaff algebriques, ser. Lecture Notes in Mathematics. Springer, 1979.

[18] J. M. Ollagnier and A. Nowicki, "Derivations of polynomial algebras without Darboux polynomials," J. Pure Appl. Algebra, vol. 212, no. 7, pp. 1626-1631, 2008.

[19] A. A. Ahmadi, M. Krstic, and P. A. Parrilo, "A globally asymptotically stable polynomial vector field with no polynomial Lyapunov function," in $C D C-E C E, 2011$, pp. 7579-7580.

[20] A. Bostan, G. Chèze, T. Cluzeau, and J.-A. Weil, "Efficient algorithms for computing rational first integrals and Darboux polynomials of planar polynomial vector fields," CoRR, vol. abs/1310.2778, 2013.

[21] Y.-K. Man, "Computing closed form solutions of first order odes using the prelle-singer procedure." J. Symb. Comput., vol. 16, no. 5, pp. 423443, 1993.

[22] Y.-K. Man and M. A. H. MacCallum, "A rational approach to the prelle-singer algorithm." J. Symb. Comput., vol. 24, no. 1, pp. 31-43, 1997.

[23] G. Cheze, "Computation of Darboux polynomials and rational first integrals with bounded degree in polynomial time," J. Complex., vol. 27, no. 2, pp. 246-262, Apr. 2011. [Online]. Available: http://dx.doi.org/10.1016/j.jco.2010.10.004

[24] J. Lasserre, Moments, Positive Polynomials and Their Applications, ser. Imperial College Press optimization series. Imperial College Press, 2009.

[25] A. Vannelli and M. Vidyasagar, "Maximal lyapunov functions and domains of attraction for autonomous nonlinear systems," Automatica, vol. 21, no. 1, pp. $69-80,1985$.

[26] A. Korobeinikov and G. C. Wake, "Global Properties of the ThreeDimensional Predator-Prey Lotka-Volterra Systems," Journal of Applied Mathematics \& Decision Sciences, vol. 3, pp. 155-162, 1999.

[27] Y. Tang, R. Yuan, and Y. Ma, "Dynamical behaviors determined by the Lyapunov function in competitive Lotka-Volterra systems," preprint, vol. 87, no. 1, Jan. 2013 